Research Paper

\title{
Prognostic Dynamic Nomogram Integrated with Inflammation-Based Factors for Non-Small Cell Lung Cancer Patients with Chronic Hepatitis B Viral Infection
}

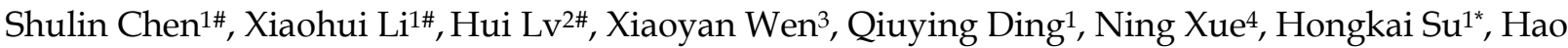 \\ Chen ${ }^{1 凶 *}$
}

1. State Key Laboratory of Oncology in South China, Collaborative Innovation Center for Cancer Medicine, Sun Yat-sen University Cancer Center, Guangzhou, 510060, P. R. China;

2. Department of Clinical Laboratory, Eighth Affiliated Hospital of Guangxi Medical University, Guigang City Pepole's Hospital, Guigang, 537100, P. R. China;

3. Department of Urology , the First Municipal Hospital of Guangzhou, Guangzhou 510180 , P. R. China;

4. Department of Clinical Laboratory, Affiliated Tumor Hospital of Zhengzhou University, Henan Tumor Hospital, Zhengzhou, 450100, P. R. China.

\#Authors contributed equally to this work.

*Authors contributed equally to this work.

$\square$ Corresponding author: Hao Chen, State Key Laboratory of Oncology in South China, Collaborative Innovation Center for Cancer Medicine, Sun Yat-sen University Cancer Center, 651 Dongfeng Road East, Guangzhou 510060, P. R. China (e-mail: chenhao@sysucc.org.cn). Tel/Fax: +86-20-8734-3438

(C) Ivyspring International Publisher. This is an open access article distributed under the terms of the Creative Commons Attribution (CC BY-NC) license (https://creativecommons.org/licenses/by-nc/4.0/). See http://ivyspring.com/terms for full terms and conditions.

Received: 2018.05.04; Accepted: 2018.09.11; Published: 2018.10.19

\begin{abstract}
Chronic inflammation plays an important role in tumor progression. The aim of this study was to develop an effective predictive dynamic nomogram integrated with inflammation-based factors to predict overall survival (OS) of non-small cell lung cancer (NSCLC) patients with chronic hepatitis B viral (HBV) infection. We retrospectively analyzed NSCLC patients with HBV infection from Sun Yat-sen University Cancer Center between 2008 and 2010. Univariate and multivariate Cox survival analyses were performed to identify prognostic factors associated with OS of patients. All of the independent prognostic factors were utilized to build the dynamic nomogram. The predictive accuracy of the dynamic nomogram was evaluated concordance index (C-index), decision curve analysis and were compared with previous reported model and traditional TNM staging system. According to the total points (TPS) by dynamic nomogram, we further stratified patients into different risk groups. A total of 203 patients were included. Multivariate Cox analysis showed TNM stage $(P=0.019)$, treatment $(P<0.001)$, C-reactive protein $(P=0.020)$ and platelet $(P=0.012)$ were independent prognostic factors of OS. The dynamic nomogram was established by involving all the factors above. The C-index of dynamic nomogram for predicting OS was 0.76 ( $95 \% \mathrm{Cl}: 0.72-0.80$ ), which was statistically higher than that of traditional TNM staging system $(0.70,95 \% \mathrm{Cl}$ : $0.66-0.74, P<0.001)$. Decision curve analysis demonstrated that the dynamic nomogram was better than the TNM staging system. The predictive accuracy of the current model keeping almost the same accuracy as previous one. Based on the total points (TPS) of dynamic nomogram, we divided the patients into 3 subgroups: low risk (TPS $\leq 107$ ), intermediate risk $(107<$ TPS $\leq 149)$, and high risk (TPS > 149). The differences of OS rates were significant in the subgroups. We propose a novel dynamic nomogram model based on inflammatory prognostic factors that is highly predictive of OS in NSCLC patients with HBV infection and outperforms the traditional TNM staging system.
\end{abstract}

Key words: dynamic nomogram, hepatitis B viral, inflammation based factors, non-small cell lung cancer, prognosis 


\section{Introduction}

Lung cancer is one of the major causes of cancer morbidity and mortality on a global scale[1]. Non-small cell lung cancer (NSCLC) accounts for $80-85 \%$ of all lung cancer cases, and its 5-year overall survival rate about $15 \%$ [2]. Chronic hepatitis B viral (HBV) infection is a serious global public problem: about two billion people have been infected with HBV worldwide[3]. However, the infection status is more severe in China, because HBV patients in China account for approximately $38 \%$ of all patients worldwide[4]. NSCLC patients with chronic HBV infection are common in the clinical setting. Previous studies have reported that chronic HBV infection was associated with poor prognosis in patients with nasopharyngeal carcinoma[5], pancreatic cancer[6], and NSCLC[7]. Thus, a distinct prognostic model is required to distinguished NSCLC patients with $\mathrm{HBV}$ infection from those without HBV infection.

Chronic HBV (CHB) infection could induce chronic inflammation in patients with $\mathrm{CHB}$ [8]. Inflammation has been confirmed to aid in the proliferation and survival of malignant cells, and it can promote tumor initiation and metastasis[9]. Previous studies have reported that serum inflammatory-based markers were significant factors for prognosis in cancer, including serum albumin (ALB)[10], serum C-reactive protein (CRP)[11], neutrophil/lymphocyte ratio (NLR)[12-13], and platelet/lymphocyte ratio (PLR)[13]. Several studies have reported that inflammation factors were biomarkers for the prognosis of NSCLC patients [14-16]. However, the effect of inflammation-based factors in NSCLC patients with HBV infection on survival is unclear.

There were four aims of the present retrospective study. First, we aimed to evaluate the prognostic significance of inflammation-based factors and clinicopathological characteristics in NSCLC patients with HBV infection. Secondly, we aimed to establish a prognostic model to be used for individual risk estimation at the point of diagnosis for NSCLC patients with HBV infection. Thirdly, we aimed to compare the inflammation-based prognostic model with traditional TNM (tumor, node, metastasis) staging. Finally, we aimed to calculate the prognosis scores of patients based on our prognostic model, and then according to those prognosis scores, the patients will be divided into a high risk group, an intermediate risk group, and a low risk group.

\section{Methods and Materials}

\section{Patients and study design}

We performed a retrospective review of NSCLC patients with chronic HBV infection. Patients first visited the Sun Yat-sen University Cancer Center (Guangzhou, China) between January 2008 and December 2010. This study was approved by the Clinical Research Ethics Committee of the Sun Yat-sen University Cancer Center, and all patients provided written informed consent at the first visit to our center. The patients included in the analysis met the following criteria: 1) NSCLC diagnosis confirmed by histopathology, were not any malignancies besides NSCLC; 2 ) all patients with HBsAg $(+)$, and excluding acute hepatitis; 3$)$ inflammation-based factors were obtained prior to anticancer treatment; 4) no liver fibrosis, steatosis and cirrhosis were detected by CT or ultrasonographic examination.

Clinical information was collected from medical records at the Cancer Center. The clinical stage of the disease was determined according to 7th edition of the AJCC TNM staging manual[17]. The clinical characteristics analyzed were gender, age, family history, smoking behavior, TNM stage, histological type, tumor size, and treatment. The potential prognostic factors included ALB, CRP, WBC, neutrophil, lymphocyte, platelet, NLR, PLR, and prognostic nutritional index (PNI).

The PNI was calculated by the following formula: albumin $(\mathrm{g} / \mathrm{L})+5 \times$ lymphocyte count $\times 10^{9} / \mathrm{L}$. Patients with a PNI $>45$ were assigned a score of 0 , and patients with a PNI $\leq 45$ were assigned a score of 1[18]. The various ratios were calculated as follows: NLR = N / L; and PLR = PLT / L, N, L, and PLT were the levels of neutrophils, lymphocytes, and platelet counts, respectively, before treatment with anticancer drugs.

The patients were followed up via clinic visits and telephone interviews. The overall survival (OS) was defined as the interval between the initial diagnosis and either death of cancer or the last follow-up. All patients were followed up until death or January 2016, if still alive. The authenticity of this article has been validated by uploading the key raw data onto the Research Data Deposit public platform (www.researchdata.org.cn), with the approval RDD number as RDDA2018000615.

\section{Statistical analysis}

Statistical analyses were performed using SPSS software, version 19.0 (SPSS Inc., Chicago, IL, USA) and $\mathrm{R}$ (version 3.4.4) for Windows. Categorical variables were classified based on clinical findings. The cut-off values of WBC and neutrophil were based on biological inflammation cutoff points in clinical application[19], and other continuous variables were transformed into categorical variables based on the cut-off values of the X-tile program[20]. The 
Kaplan-Meier curves were used to calculate the survival rate, and the Log-rank test was used to compare them. Univariate analysis was performed to assess the significance of clinical and pathological characteristics. The Cox proportional hazards regression was used for multivariate analysis. All variables with $\mathrm{P}$ values less than 0.05 in the multivariable model were used to develop a dynamic prediction nomogram model. The performance of the dynamic prediction nomogram model was measured by the concordance index (C-index), and validated using 1000 bootstrap re-samplings. Comparison of the prediction accuracy between the dynamic prediction nomogram model, reported model[21] and traditional TNM staging systems in NSCLC patients with HBV infection were performed using the C-index[22] and decision curve analysis[23]. The difference was considered statistically significant when $\mathrm{p}<0.05$.

\section{Results}

\section{Patient characteristics}

In this study, a total of 203 NSCLC patients with HBV infection were enrolled. the median age was 56 years (range 27-82 years); there were 155 (76.4\%) were males and $48(23.6 \%)$ females. At the time of the last follow-up, the median OS was 33 months. The 3- and 5 -year OS rates for patients were $47.3 \%$ and $32.0 \%$, respectively. The demographic and clinical characteristics of the 203 patients are summarized in Table 1 . The following cut-off values for continuous variables were obtain using the X-tile program: age (42 years), tumor size $(6.0 \mathrm{~cm})$, ALB $(37.6 \mathrm{~g} / \mathrm{L}), \mathrm{CRP}$ (9.5 $\mathrm{mg} / \mathrm{L}), \quad$ lymphocyte $\left(1.8 \times 10^{\wedge} / \mathrm{L}\right), \quad$ PLT

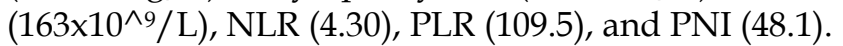

\section{Univariate analysis and multivariate Cox proportional hazards regression analysis of the overall survival}

The univariate analysis revealed that age, TNM stage, size, treatment, ALB, CRP, WBC, neutrophil, PLT, NLR, PLR, and PNI were associated with OS $(P<0.05$, Table 2$)$. Then the prognostic factors that were significantly associated with $O S$ in the univariate analyses were entered into the multivariate Cox proportional hazards regression analysis of OS. The multivariate analysis showed that TNM stage $(\mathrm{HR}=1.40,95 \% \mathrm{CI}=1.06-1.86, P=0.019)$, treatment $(\mathrm{HR}=1.76, \quad 95 \% \quad \mathrm{CI}=1.28-2.40, \quad P<0.001), \quad \mathrm{CRP}$ $(\mathrm{HR}=1.76,95 \% \mathrm{CI}=1.09-2.84, P=0.020)$, and PLT $(\mathrm{HR}=0.43, \quad 95 \% \quad \mathrm{CI}=0.22-0.83, \quad P=0.012) \quad$ were independently associated with OS. A forest plot shows the hazard ratios and $95 \%$ confidence intervals for OS according to the Cox proportional hazards regression analysis (Figure 1).
Table 1. Patient demographics and clinical characteristics

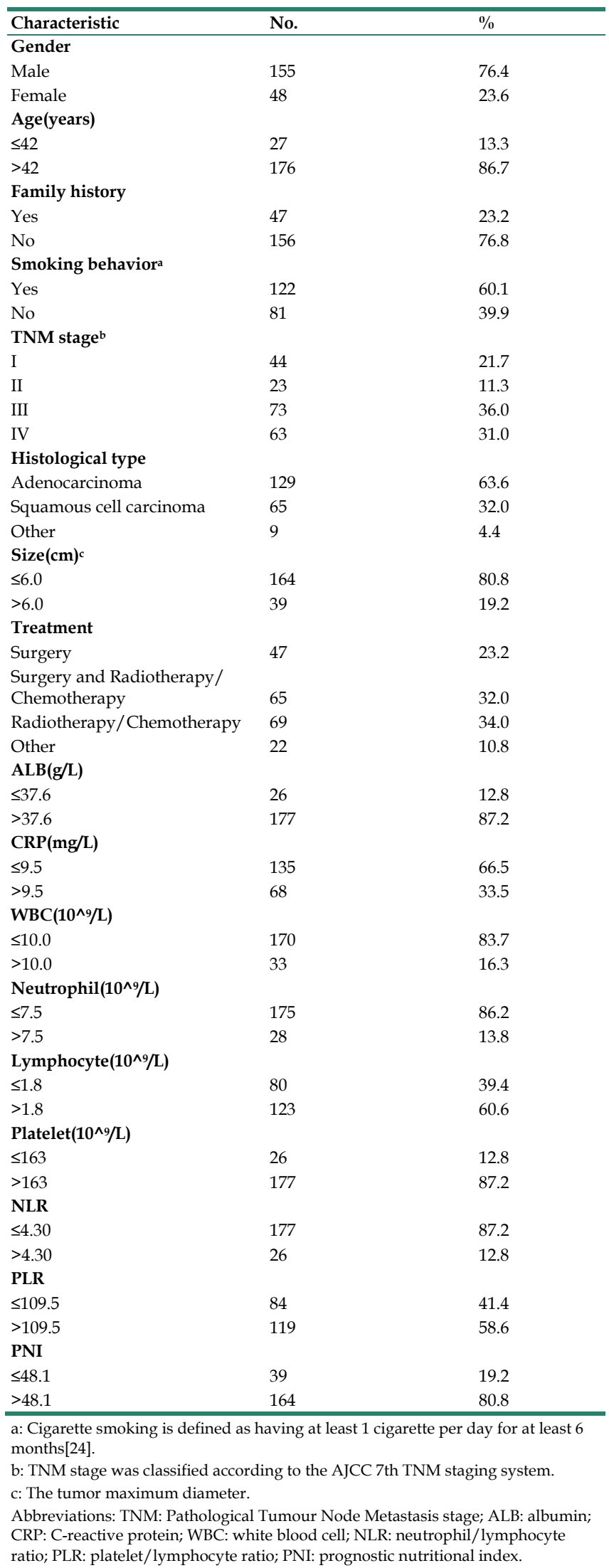


Table 2. Univariate and multivariate Cox proportional hazards regression analysis for OS

\begin{tabular}{|c|c|c|c|c|}
\hline \multirow[t]{2}{*}{ Variable } & \multicolumn{2}{|c|}{ Univariate analysis } & \multicolumn{2}{|c|}{ Multivariate analysis } \\
\hline & HR (95\% CI) & $P$ & HR (95\% CI) & $P$ \\
\hline \multicolumn{5}{|l|}{ Gender } \\
\hline Male & Reference & & \multirow[b]{2}{*}{ - } & \multirow[b]{2}{*}{ - } \\
\hline Female & $0.85(0.53-1.36)$ & 0.495 & & \\
\hline \multicolumn{5}{|l|}{ Age(years) } \\
\hline$\leq 42$ & Reference & & \multirow[t]{2}{*}{-} & \multirow[t]{2}{*}{-} \\
\hline$>42$ & $0.32(0.19-0.53)$ & $<0.001$ & & \\
\hline \multicolumn{5}{|l|}{ Family history } \\
\hline Yes & Reference & & \multirow{2}{*}{ - } & \multirow{2}{*}{ - } \\
\hline No & $0.92(0.58-1.46)$ & 0.735 & & \\
\hline \multicolumn{5}{|c|}{ Smoking behavior } \\
\hline Yes & Reference & & \multirow[b]{2}{*}{ - } & \multirow[b]{2}{*}{ - } \\
\hline No & $1.03(0.69-1.53)$ & 0.901 & & \\
\hline \multicolumn{5}{|l|}{ TNM stage } \\
\hline I & Reference & & \multirow{4}{*}{$1.40(1.06-1.86)$} & \\
\hline II & $1.08(0.40-2.87)$ & 0.885 & & 0.019 \\
\hline III & $2.75(1.43-5.30)$ & 0.003 & & \\
\hline IV & $6.61(3.35-12.72)$ & $<0.001$ & & \\
\hline Histological type & & & & \\
\hline Adenocarcinoma & Reference & & & \\
\hline $\begin{array}{l}\text { Squamous } \\
\text { cell carcinoma }\end{array}$ & $0.89(0.58-1.38)$ & 0.603 & - & - \\
\hline Other & $1.68(0.68-4.17)$ & 0.265 & & \\
\hline $\operatorname{Size}(\mathrm{cm})$ & & & & \\
\hline$\leq 6.0$ & Reference & & 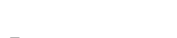 & 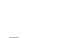 \\
\hline$>6.0$ & $2.35(1.50-3.70)$ & $<0.001$ & - & - \\
\hline Treatment & & & & \\
\hline Surgery & Reference & & & \\
\hline Surgery and & & & & \\
\hline $\begin{array}{l}\text { Radiotherapy/ } \\
\text { Chemotherapy }\end{array}$ & $0.91(0.47-1.74)$ & 0.772 & $1.76(1.28-2.40)$ & $<0.001$ \\
\hline $\begin{array}{l}\text { Radiotherapy/ } \\
\text { Chemotherapy }\end{array}$ & $3.80(2.11-6.84)$ & $<0.001$ & & \\
\hline Other & $6.99(3.47-14.06)$ & $<0.001$ & & \\
\hline $\operatorname{ALB}(g / L)$ & & & & \\
\hline$\leq 37.6$ & Reference & & 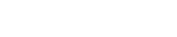 & 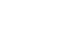 \\
\hline$>37.6$ & $0.40(0.25-0.66)$ & $<0.001$ & - & - \\
\hline $\mathrm{CRP}(\mathrm{mg} / \mathrm{L})$ & & & & \\
\hline$\leq 9.5$ & Reference & & $1.76(1.09-2.84)$ & 0.020 \\
\hline$>9.5$ & $2.70(1.81-4.03)$ & $<0.001$ & & \\
\hline WBC $\left(10^{\wedge} 9 / \mathrm{L}\right)$ & & & & \\
\hline$\leq 10.0$ & Reference & & & \\
\hline$>10.0$ & $1.92(1.17-3.14)$ & 0.009 & - & - \\
\hline Neutrophil(10^9/ & & & & \\
\hline$\leq 7.5$ & Reference & & 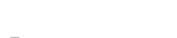 & 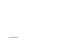 \\
\hline$>7.5$ & $2.66(1.61-4.40)$ & $<0.001$ & - & - \\
\hline Lymphocyte(/10 & & & & \\
\hline$\leq 1.8$ & Reference & & & \\
\hline$>1.8$ & $0.80(0.54-1.19)$ & 0.268 & - & - \\
\hline Platelet $\left(10^{\wedge} 9 / \mathrm{L}\right)$ & & & & \\
\hline$\leq 163$ & Reference & & $0.43(0.22-0.83)$ & 0.012 \\
\hline$>163$ & $0.50(0.30-0.84)$ & 0.009 & & \\
\hline NLR & & & & \\
\hline$\leq 4.30$ & Reference & & 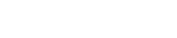 & \\
\hline$>4.30$ & $3.30(1.96-5.55)$ & $<0.001$ & - & - \\
\hline PLR & & & & \\
\hline$\leq 109.5$ & Reference & & & \\
\hline$>109.5$ & $1.57(1.04-2.38)$ & 0.034 & - & - \\
\hline PNI & & & & \\
\hline$\leq 48.1$ & Reference & & & \\
\hline$>48.1$ & $0.40(0.26-0.63)$ & $<0.001$ & - & - \\
\hline
\end{tabular}

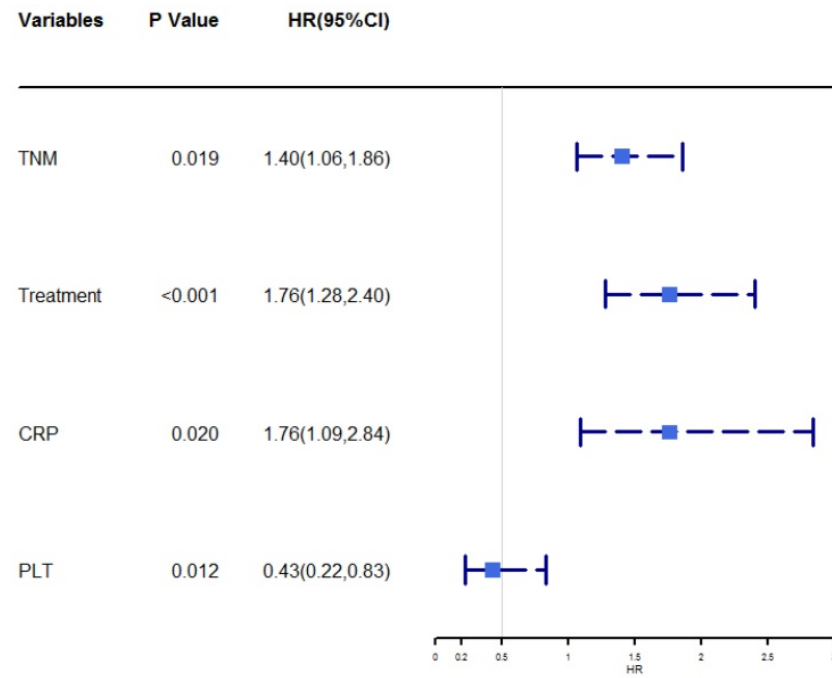

Figure 1. Forest plot showed the hazard ratio and $95 \%$ confidence interval for over survival according to the Cox proportional hazards regression analysis.

\section{Construction and validation of dynamic prediction nomogram}

All of the independent predictors of OS in multivariate Cox proportional hazards regression analysis were integrated into the dynamic prediction nomogram model, and the 3- and 5-year survival probability were graphically computed based on the unique characteristics of an individual patient (Figure. 2). The models include TNM stage, CRP, PLT at diagnosis, and treatment. The C-index of the dynamic prediction nomogram model was $0.76 \quad(95 \%$ $\mathrm{CI}=0.72-0.80)$.

Each prognostic factor has a number of risk points, which can be obtained by drawing a vertical line straight up from the prognostic factor's corresponding value to the axis with "Points". To determine the 3- and 5-year OS probability of a specific patient from the "Total Points" which is the sum of the risk points, a vertical line can be drawn towards the axis labeled "3- and 5-Year Overall Survival Probability". For example (Figure. 2), we will consider a patient had a TNM stage III (31 points) with a CRP level of $118.7 \mathrm{mg} / \mathrm{L}$ (80 points), a PLT of $394 \times 10^{\wedge} 9 / \mathrm{L}$ (16.5 points), and the patient has undergone surgery (6.5 points). Thus, the sum of the total risk points was 134, and a vertical line can be drawn down to the "3- and 5-year survival probability" axis. For this patient, the 3-year survival probability is $35.6 \%$, and the 5-year survival probability is $20.0 \%$. 

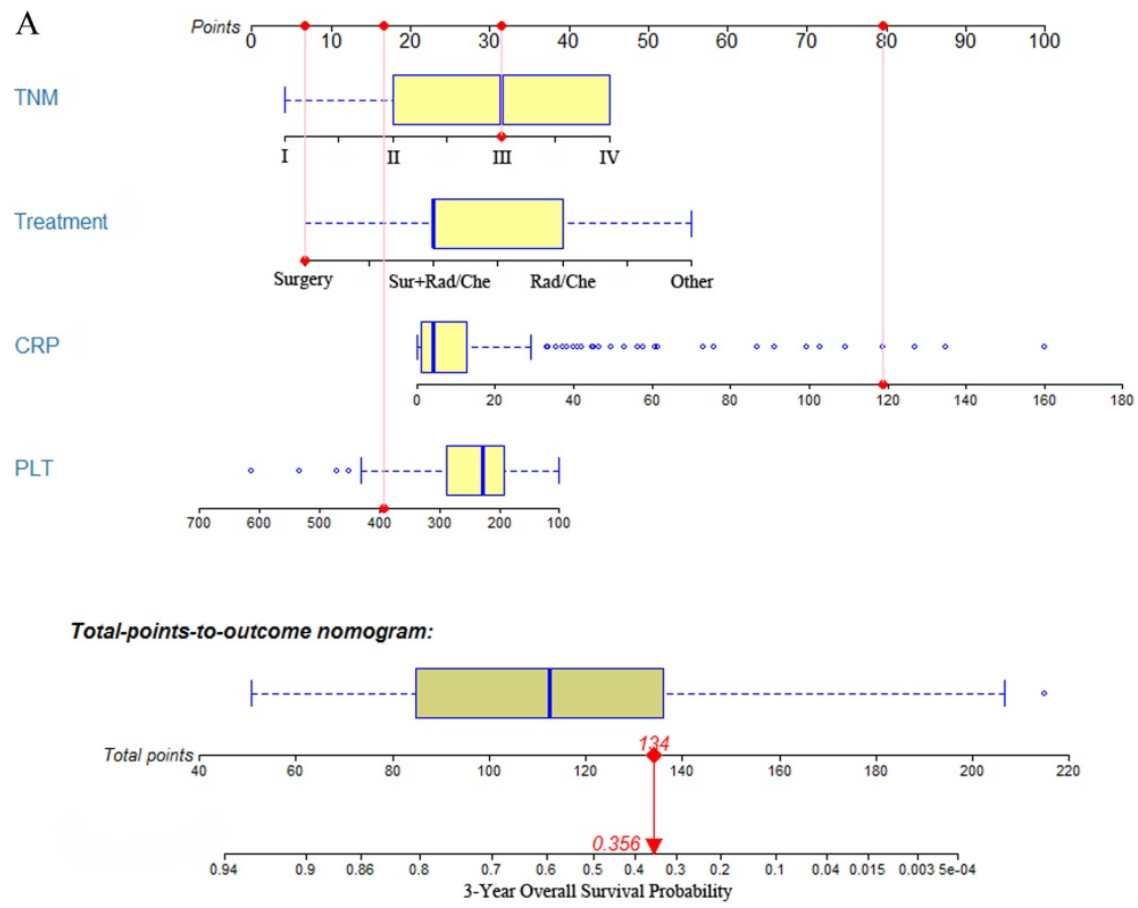

B

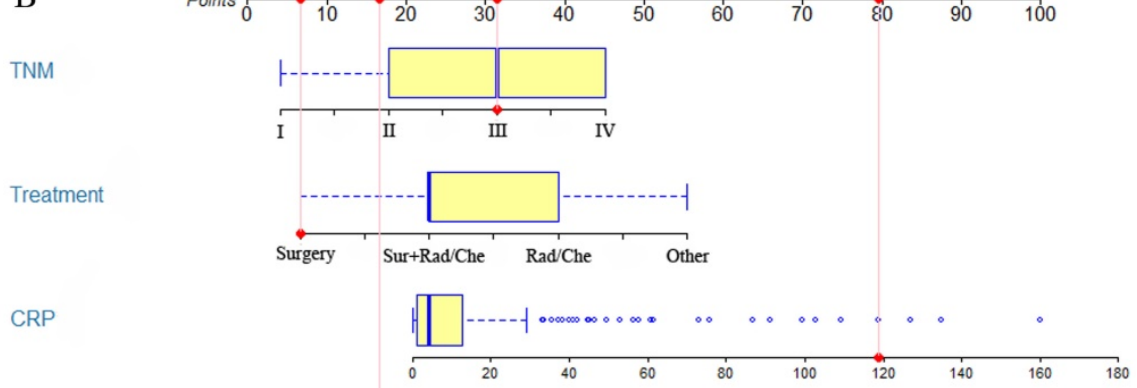

PLT
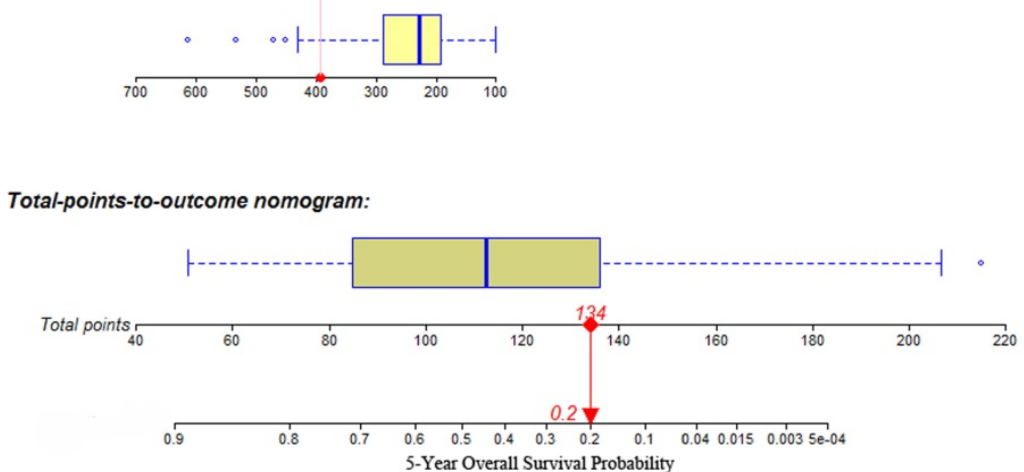

Figure 2. The 3- and 5-year overall survival probability is calculated by taking the sum of the risk points, according to TNM stage, treatment, CRP, and PLT. For each parameter, the number of associated risk points can be determined by drawing a vertical line straight up from the covariate's corresponding value to the axis with risk points $(0$ to 100$)$. In order to determine the 5 -year overall survival probability, a vertical line is drawn intersecting the "Total points" with the corresponding survival line.

\section{Comparison of the predictive accuracy between current dynamic nomogram, reported nomogram and conventional staging systems}

Comparison of the predictive accuracy of current nomogram model with reported nomogram model and conventional staging systems were done using C-index and decision curve analysis for 3- and 5-year survival. A larger C-index and area under the curve of the decision curve indicates a more accurate prognostic stratification. The $\mathrm{C}$-index of the current nomogram model was $0.76(95 \% \mathrm{CI}=0.72-0.80)$, 
which was significantly better than the TNM staging systems $0.70(95 \% \mathrm{CI}=0.66-0.74$, Table 3$)$. There was no statistical difference between the current model and reported model $(0.77,95 \% \mathrm{CI}=0.63-0.81)$. Decision curve analysis for 3- and 5-year survival (Figure. 3A, 3B, respectively) revealed that the predictive accuracy of the current nomogram (black dotted line) had a higher net benefit than the TNM staging systems (green dotted line). The predictive accuracy of the current nomogram model keeping almost the same accuracy as previous reported one (red dotted line).
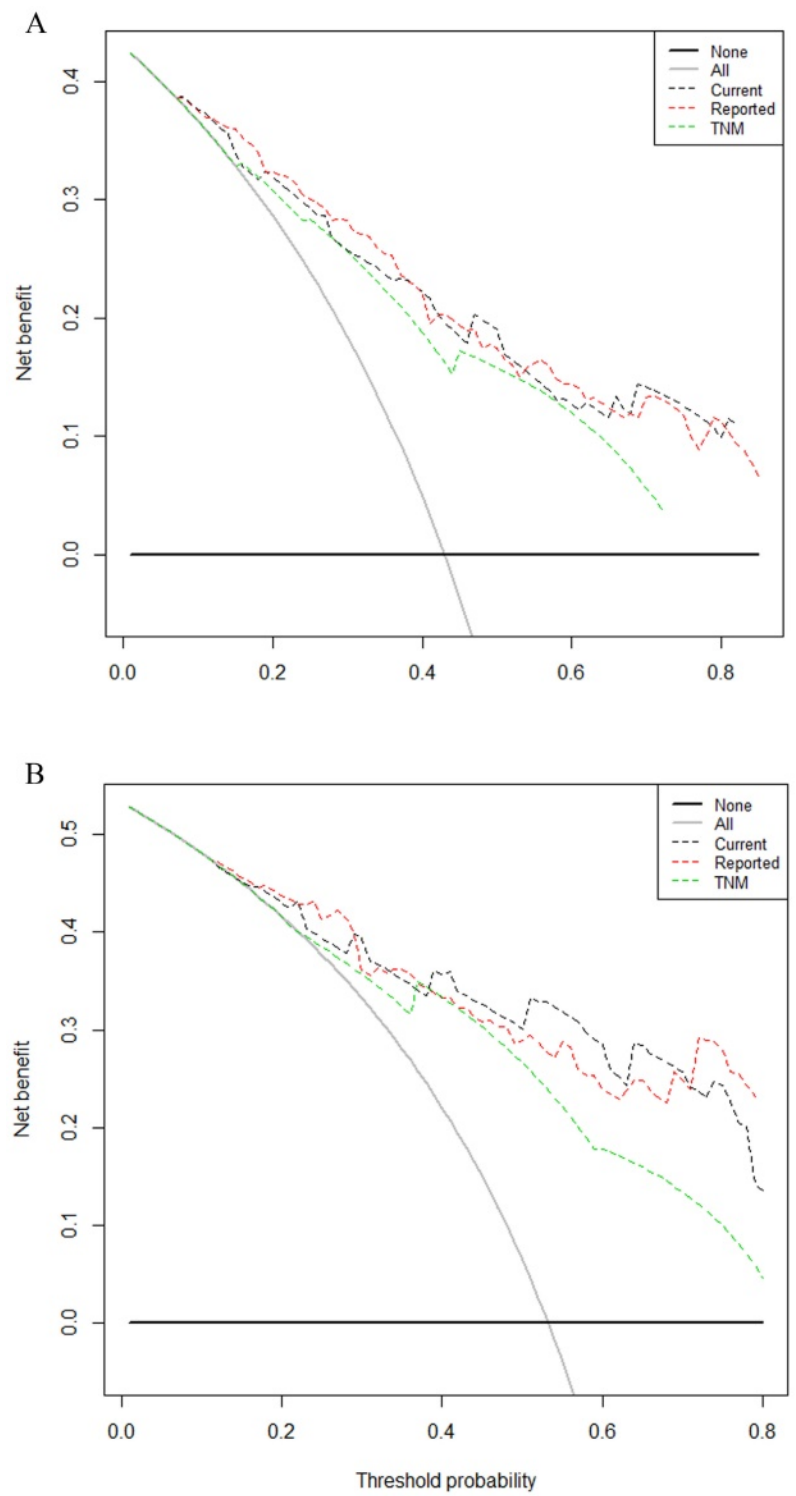

Figure 3. The decision curve analysis for 3, 5-year survival. A: Decision curve analysis for 3-year survival; B: Decision curve analysis for 5-year survival. Black dotted line: current nomogram; Green dotted line: TNM staging system; Red dotted line: reported nomogram. $Y$-axis indicates net benefit, calculated by summing the benefits (true positives) and subtracting the harms (false positives). The straight line represents the assumption that all patients will die, and the horizontal line represents the assumption that no patients will die.

\section{Performance of the dynamic nomogram in stratifying patient risk}

All patients were further stratified by the total points (TPS) of the dynamic prediction nomogram model for OS analysis. We divided the patients into three groups: low risk (total points [TPS] $\leq 107,90$ patients), intermediate risk $(107<$ TPS $\leq 149,89$ patients) and high risk (TPS > 149, 24 patients). The Kaplan-Meier plot showing the OS for patients of the three subgroups indicates that the high risk group is linked to a poor prognosis (Figure. 4). The differences between OS rates were significant $(P<0.001)$.

Table 3. The C-index of current nomogram, reported nomogram and TNM stage for prediction of OS*

\begin{tabular}{lll}
\hline Factor & C-index (95\% CI) & $p$ \\
\hline Current nomogram* & $0.76(0.72-0.80)$ & \\
Reported nomogram & $0.77(0.73-0.81)$ & \\
TNM stage & $0.70(0.66-0.74)$ & \\
Current nomogram vs TNM stage & $<0.001$ \\
Reported nomogram vs TNM stage & $<0.001$ \\
Current nomogram vs Reported nomogram & 0.67 \\
\hline * Current nomogram: including eight risk factors (age, TNM, treatment, CRP and \\
PLT).
\end{tabular}

\section{Discussion}

In this study, we utilized the advantages of the ability of dynamic nomogram with integration of inflammation-based factors and clinicopathological characteristics to establish an effective survival prognostic model for NSCLC patients with HBV infection. The dynamic nomogram model has better predictive accuracy and discriminative ability. In addition, it can be used for individual risk estimation at the point of diagnosis for NSCLC patients with HBV infection, which can aid in the decision-making process in clinical practice.

The dynamic nomogram included TNM stage, treatment, CRP, and PLT. Currently, the Union Internationale Contre le Cancer/American Joint Cancer Committee (UICC/AJCC) TNM staging system is widely used as a guide for estimating prognosis, and it is the basis for treatment decisions of various solid tumors. However, the classification is only based on the anatomical extent of the disease, which doesn't fully reflect the biological heterogeneity of NSCLC patients, and other risk factors were not taken into account in the systems. Thus, the traditional TNM staging system may not accurately predict the OS for NSCLC patients. Integration of the TNM staging system with other risk factors could be highly predictive of OS in NSCLC patients with HBV infection. Interestingly, our results indicated that the C-index of the current dynamic 
nomogram (0.76) was higher than the traditional TNM staging system (0.70). Also, the decision curve analysis for 3- and 5-year survival revealed a better predictive accuracy of the dynamic nomogram than TNM staging system. We previously had reported that a nomogram model for NSCLC patients with chronic hepatitis B viral infection based on the prognostic factors of liver function indicators[21]. Considering the systemic inflammation has been linked with poor prognosis in patients with cancer. So, in this study, we only focus on the prognosis of inflammation-based factors for NSCLC with HBV infection, and propose a nomogram model for NSCLC patients with HBV infection. We compared current nomogram model with previous model, the predictive accuracy of the current nomogram keeping almost the same accuracy as previous one. The C-index of current nomogram was 0.76 , and previous reported nomogram was 0.77 . There was no statistical difference between the two nomogram models. But the current nomogram included less prognostic factors (4 vs 8 ). So current nomogram model maybe more convenient for clinical applications.

CRP is an acute-phase protein that plays important roles in protecting tissue from inflammation, and it is a simple and reproducible systemic inflammation marker. Many studies have reported that the level of CRP is related to the prognosis of various cancers[13-14, 25-26]. CRP production can be caused by interleukin (IL)-6. IL-6 as an important proinflammatory cytokine that could promote cancer growth and metastasis through Janus kinase (JAK)-signal transducer and activator of transcription (STAT) 3 activation. Thus, CRP could be a marker of IL-6/JAK/STAT3-induced tumor progression[27]. PLT is associated with systemic inflammation and cancerous diseases, which have emerged as central players in the progression of cancer and local responses to tumor growth[28]. Within the blood circulation system, PLT could protect cancer cells from immune elimination and promote their arrest in the endothelium. This supports the establishment of secondary lesions, which contributes to cancer cell survival and migration[29]. Our results were consistent with previously reported findings.

The univariate analysis revealed that ALB, NLR, PLR, and PNI were all associated with OS, but the multivariate analysis results indicated that they were not independently associated with OS. These results were contrary to previously reported findings [17, 30-35]. A possible explanation is that our continuous variables were transformed into high and low groups based on the cut-off values of the X-tile program, which was different from other reports. A second possible explanation is that previous studies have reported that chronic HBV infection was associated with poor prognosis in patients with NSCLC[7], so NSCLC patients with HBV infection have different clinicopathological characteristics compared to those without HBV infection. This may be the most important reason that leads to differing results from other reports.

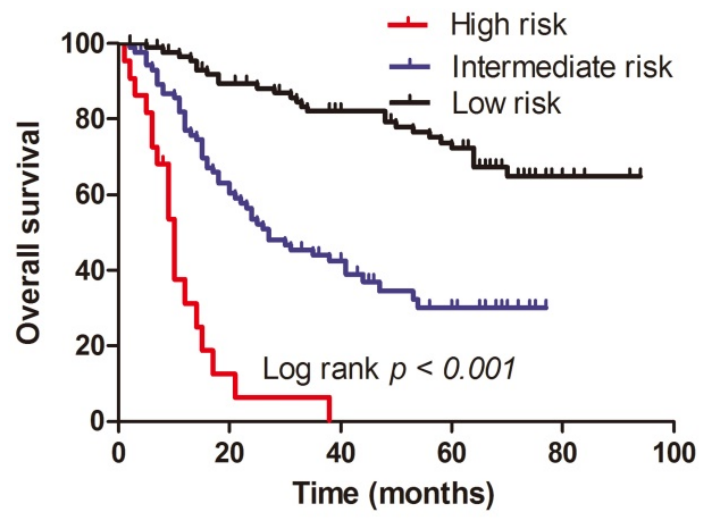

\begin{tabular}{|l|c|c|c|}
\hline & Low risk & Intermediate risk & High risk \\
\hline No. (\%) & $90(44.3)$ & $89(43.9)$ & $24(11.8)$ \\
\hline Median OS, months & 63 & 21 & 9 \\
\hline HR $(95 \%$ CI) & Reference & $3.69(2.28-5.99)$ & $17.75(9.37-33.63)$ \\
\hline Log rank $P$ value & Reference & $<0.001$ & $<0.001$ \\
\hline
\end{tabular}

Figure 4. Kaplan-Meier curves for three subgroups based on the predictor from dynamic nomogram model.

We further divided the patients into three risk groups based on TPS. The survival curves separated very well in the patients $(P<0.001)$, and our results indicated that a higher TPS was associated with a shorter OS in NSCLC patients with HBV infection. Consequently, patients with high TPS in high-risk groups should receive special attention and active surveillance.

There were certain limitations in our study. First, this study was typical of retrospective analyses, so the retrospective nature of this study cannot exclude all potential biases. Second, many studies were adopted major three methods to determine the cut-off values of prognostic factors: a: median[36]; b: X-tile tool[20, 37]; c: ROC method[38-39]. The cut-off values of each method were determined by the analyzed patients data, which leading different analyzed patients data had different cut-off values in the same prognostic factor. In our study, the cut-off values of some prognostic factors were determined by using X-tile tool. The best way to solve this problem was to validate the results using external data. Thirdly, the database used to generate the dynamic nomogram consisted of patient data from a single cancer center, and the sample sizes were small. Further larger scale and multi-center studies should be performed to 
externally validate the dynamic nomogram and to verify whether our results were suitable for the general population. At last, this study only focused on the impact of inflammation-based prognostic factors on the prognosis of NSCLC patients with HBV infection; other traditional prognostic factors, such as cytokeratin 19 fragment (CYFRA21-1), carcinoembryonic antigen (CEA), squamous cell carcinoma antigen (SCC), epidermal growth factor receptor (EGFR), and CA125 were not analyzed in this study[40-43]. Furthermore, previous studies have reported that serum HBV-DNA at diagnosis is an independent and significant prognostic factor in hepatocellular carcinoma[44]. However, measuring HBV-DNA was not common practice in our cancer center. Thus, whether HBV DNA levels were associated with the prognosis in NSCLC patients with HBV infection should be further explored.

In summary, the dynamic nomogram was a simple and inexpensive prognostic tool for NSCLC patients with HBV infection, and it has better predictive accuracy and discriminative ability. It could aid clinicians in the decision-making process in clinical practice.

\section{Acknowledgments}

This work was supported by the Natural Science Foundation of Guangdong Province (2018A0303136 22). We thank the staff at the Director of Clinical Laboratories, Sun Yat-sen University Cancer Center for providing support on research conditions in this study.

\section{Author contributions}

All authors contributed to this manuscript, including conception and design (SLC, XHL, HL), acquisition of data (QYD), analysis and interpretation of data (XYW), material support (QYD), study supervision (HSK, HC), and writing, review and revision of the manuscript (SLC, XHL, NX, HKS, HC).

\section{Competing Interests}

The authors have declared that no competing interest exists.

\section{References}

1. Siegel RL, Miller KD, PhD AJD. Cancer statistics, 2017. Ca A Cancer Journal for Clinicians. 2017; 67: 7-30.

2. Pao W, Girard N. New driver mutations in non-small-cell lung cancer. Lancet Oncology. 2011; 12: 175-80.

3. Ott JJ, Stevens GA, Groeger J, Wiersma ST. Global epidemiology of hepatitis B virus infection: new estimates of age-specific HBsAg seroprevalence and endemicity. Vaccine. 2012; 30: 2212-9.

4. Zheng X, Wang J, Yang D. Antiviral therapy for chronic hepatitis B in China. Med Microbiol Immunol. 2015; 204: 115-20.

5. Liu X, Li X, Jiang N, Lei Y, Tang LL, Chen L, et al. Prognostic value of chronic hepatitis $\mathrm{B}$ virus infection in patients with nasopharyngeal carcinoma: analysis of 1301 patients from an endemic area in China. cancer. 2014; 120: 68.
6. Wei XL, Qiu MZ, Chen WW, Jin Y, Ren C, Wang F, et al. The status of HBV infection influences metastatic pattern and survival in Chinese patients with pancreatic cancer. Journal of Translational Medicine. 2013; 11: 249.

7. Peng JW, Liu DY, Lin GN, Xiao JJ, Xia ZJ. Hepatitis B Virus Infection Is Associated with Poor Prognosis in Patients with Advanced Non Small Cell Lung Cancer. Asian Pac J Cancer Prev. 2015; 16: 5285-8.

8. Xie Y. Hepatitis B Virus-Associated Hepatocellular Carcinoma. Adv Exp Med Biol. 2017; 1018: 11-21.

9. Mantovani A, Allavena P, Sica A, Balkwill F. Cancer-related inflammation. Nature. 2008; 454: 436-44.

10. Saito H, Kono $Y$, Murakami $Y$, Shishido $Y$, Kuroda $H$, Matsunaga $T$, et al Postoperative Serum Albumin is a Potential Prognostic Factor for Older Patients with Gastric Cancer. Yonago Acta Med. 2018; 61: 72-8.

11. Yanagisawa M, Gingrich AA, Judge S, Li CS, Wang N, Thorpe SW, et al. Serum C-reactive Protein and Neutrophil/Lymphocyte Ratio After Neoadjuvant Radiotherapy in Soft Tissue Sarcoma. Anticancer Res. 2018; 38: 1491-7.

12. Kasymjanova G, MacDonald N, Agulnik JS, Cohen V, Pepe C, Kreisman H, et al. The predictive value of pre-treatment inflammatory markers in advanced non-small-cell lung cancer. Curr Oncol. 2010; 17: 52-8.

13. Li JP, Chen SL, Liu XM, He X, Xing S, Liu YJ, et al. A Novel Inflammation-Based Stage (I Stage) Predicts Overall Survival of Patients with Nasopharyngeal Carcinoma. Int J Mol Sci. 2016; 17.

14. Tomita M, Ayabe T, Maeda R, Nakamura K. Combination of Advanced Lung Cancer Inflammation Index and C-Reactive Protein Is a Prognostic Factor in Patients With Operable Non-Small Cell Lung Cancer. World J Oncol. 2017; 8: $175-9$

15. Tong YS, Tan J, Zhou XL, Song YQ, Song YJ. Systemic immune-inflammation index predicting chemoradiation resistance and poor outcome in patients with stage III non-small cell lung cancer. J Transl Med. 2017; 15: 221.

16. Tomita M, Ayabe T, Nakamura K. The advanced lung cancer inflammation index is an independent prognostic factor after surgical resection in patients with non-small-cell lung cancer. Interact Cardiovasc Thorac Surg. 2018; 26: 288-92.

17. Edge SB, Compton CC. The American Joint Committee on Cancer: the 7th edition of the AJCC cancer staging manual and the future of TNM. Ann Surg Oncol. 2010; 17: 1471-4

18. Buzby GP, Mullen JL, Matthews DC, Hobbs CL, Rosato EF. Prognostic nutritional index in gastrointestinal surgery. Am J Surg. 1980; 139: 160-7.

19. Schernberg A, Blanchard P, Chargari C, Ou D, Levy A, Gorphe P, et al. Leukocytosis, prognosis biomarker in locally advanced head and neck cancer patients after chemoradiotherapy. Clin Transl Radiat Oncol. 2018; 12: 8-15.

20. Camp RL, Dolledfilhart M, Rimm DL. X-Tile A New Bio-Informatics Tool for Biomarker Assessment and Outcome-Based Cut-Point Optimization. Clinical Cancer Research An Official Journal of the American Association for Cancer Research. 2004; 10: 7252-9.

21. Chen S, Lai Y, He Z, Li J, He X, Shen R, et al. Establishment and validation of a predictive nomogram model for non-small cell lung cancer patients with chronic hepatitis B viral infection. Journal of Translational Medicine. 2018; 16: 116.

22. Harrell FE, Jr., Lee KL, Califf RM, Pryor DB, Rosati RA. Regression modelling strategies for improved prognostic prediction. Stat Med. 1984; 3: 143-52.

23. Vickers AJ, Elkin EB. Decision curve analysis: a novel method for evaluating prediction models. Med Decis Making. 2006; 26: 565-74

24. Lai H, Liu Y, Zhou M, Shi T, Zhou Y, Weng S, et al. Combined effect of silica dust exposure and cigarette smoking on total and cause-specific mortality in iron miners: a cohort study. Environmental Health. 2018; 17: 46.

25. Takamatsu K, Mizuno R, Omura M, Morita S, Matsumoto K, Shinoda K, et al. Prognostic Value of Baseline Serum C-Reactive Protein Level in Intermediate-Risk Group Patients With Metastatic Renal-Cell Carcinoma Treated by First-Line Vascular Endothelial Growth Factor-Targeted Therapy. Clin Genitourin Cancer. 2018

26. Zhou B, Shu B, Yang J, Liu J, Xi T, Xing Y. C-reactive protein, interleukin-6 and the risk of colorectal cancer: a meta-analysis. Cancer Causes Control. 2014; 25 : 1397-405.

27. $\mathrm{Yu} \mathrm{H}$, Lee H, Herrmann A, Buettner R, Jove R. Revisiting STAT3 signalling in cancer: new and unexpected biological functions. Nature Reviews Cancer. 2014; 14: 736-46.

28. Klement GL, Yip TT, Cassiola F, Kikuchi L, Cervi D, Podust V, et al. Platelets actively sequester angiogenesis regulators. Blood. 2009; 113: 2835-42.

29. Gay LJ, Felding-Habermann B. Contribution of platelets to tumour metastasis. Nat Rev Cancer. 2011; 11: 123-34.

30. Minami S, Thara S, Kim SH, Yamamoto S, Komuta K. Lymphocyte to Monocyte Ratio and Modified Glasgow Prognostic Score Predict Prognosis of Lung Adenocarcinoma Without Driver Mutation. World J Oncol. 2018; 9: 13-20.

31. Ikeda S, Yoshioka H, Ikeo S, Morita M, Sone N, Niwa T, et al. Serum albumin level as a potential marker for deciding chemotherapy or best supportive care in elderly, advanced non-small cell lung cancer patients with poor performance status. BMC cancer. 2017; 17: 797.

32. Koh YW, Lee HW. Prognostic impact of C-reactive protein/albumin ratio on the overall survival of patients with advanced nonsmall cell lung cancers receiving palliative chemotherapy. Medicine (Baltimore). 2017; 96: e6848.

33. Diem S, Schmid S, Krapf M, Flatz L, Born D, Jochum W, et al. Neutrophil-to-Lymphocyte ratio (NLR) and Platelet-to-Lymphocyte ratio 
(PLR) as prognostic markers in patients with non-small cell lung cancer (NSCLC) treated with nivolumab. Lung cancer. 2017; 111: 176.

34. Hong S, Zhou T, Fang W, Xue C, Hu Z, Qin T, et al. The prognostic nutritional index (PNI) predicts overall survival of small-cell lung cancer patients. Tumour Biol. 2015; 36: 3389-97.

35. Qiu C, Qu X, Shen H, Zheng C, Zhu L, Meng L, et al. Evaluation of Prognostic Nutritional Index in Patients Undergoing Radical Surgery with Nonsmall Cell Lung Cancer. Nutr Cancer. 2015; 67: 741-7.

36. Zhu GS, Tian SB, Wang H, Ma MG, Liu Y, Du HS, et al. Preoperative Neutrophil Lymphocyte Ratio and Platelet Lymphocyte Ratio Cannot Predict Lymph Node Metastasis and Prognosis in Patients with Early Gastric Cancer: a Single Institution Investigation in China. Current Medical Science. 2018; 38 : 78-84.

37. Guan X, Wang Y, Hu H, Zhao Z, Jiang Z, Liu Z, et al. Reconsideration of the optimal minimum lymph node count for young colon cancer patients: a population-based study. BMC cancer. 2018; 18: 623.

38. Li XH, Chang $\mathrm{H}, \mathrm{Xu} B \mathrm{BQ}$, Tao $\mathrm{YL}$, Gao J, Chen $\mathrm{C}$, et al. An inflammatory biomarker-based nomogram to predict prognosis of patients with nasopharyngeal carcinoma: an analysis of a prospective study. Cancer Medicine. 2016; 6: 310-9.

39. Won YW, Joo J, Yun T, Lee GK, Han JY, Kim HT, et al. A nomogram to predict brain metastasis as the first relapse in curatively resected non-small cell lung cancer patients. Lung cancer. 2015; 88: 201-7.

40. Romero-Ventosa EY, Blanco-Prieto S, Gonzalez-Pineiro AL Rodriguez-Berrocal FJ, Pineiro-Corrales G, Paez de la Cadena M. Pretreatment levels of the serum biomarkers CEA, CYFRA 21-1, SCC and the soluble EGFR and its ligands EGF, TGF-alpha, HB-EGF in the prediction of outcome in erlotinib treated non-small-cell lung cancer patients. Springerplus. 2015; 4: 171.

41. Mizuguchi S, Nishiyama N, Iwata T, Nishida T, Izumi N, Tsukioka T, et al. Serum Sialyl Lewis $\mathrm{x}$ and cytokeratin 19 fragment as predictive factors for recurrence in patients with stage I non-small cell lung cancer. Lung cancer. 2007; 58: 369-75

42. Vinolas N, Molina R, Fuentes R, Bover I, Rifa J, Moreno V, et al. Tumor markers (CEA, CA 125, CYFRA 21.1, SCC and NSE) in non small cell lung cancer (NSCLC) patients as an aid in histological diagnosis and prognosis: Comparison with the main clinical and pathological prognostic factors. Tumour Biology the Journal of the International Society for Oncodevelopmental Biology \& Medicine. 2003; 29: 209-18.

43. Baek AR, Seo HJ, Lee JH, Park SW, Jang AS, Paik SH, et al. Prognostic value of baseline carcinoembryonic antigen and cytokeratin 19 fragment levels in advanced non-small cell lung cancer. Cancer Biomark. 2018.

44. Ohkubo K, Kato Y, Ichikawa T, Kajiya Y, Takeda Y, Higashi S, et al. Viral load is a significant prognostic factor for hepatitis B virus-associated hepatocellular carcinoma. cancer. 2002; 94: 2663-8. 